01

\title{
Контактные свойства градиентных материалов с высоким показателем градиентности
}

\author{
(C) Я.А. Ляшенко, ${ }^{1,2}$ В.Л. Попов ${ }^{1,3,4}$ \\ ${ }^{1}$ Берлинский технический университет, \\ 10623 Берлин, Германия \\ ${ }^{2}$ Сумский государственный университет, \\ 40007 Сумы, Украина \\ ${ }^{3}$ Национальный исследовательский Томский государственный университет, \\ 634050 Томск, Россия \\ ${ }^{4}$ Институт фризики прочности и материаловедения, \\ 634055 Томск, Россия \\ e-mail: i.liashenko@tu-berlin.de
}

Поступило в Редакцию 25 мая 2021 г.

В окончательной редакции 1 июля 2021 г.

Принято к публикации 5 июля 2021 г.

Исследован адгезионный контакт между несжимаемым параболическим индентором и полупространством, модуль упругости которого является степенной функцией глубины. Приведена диаграмма, на которой указаны области, соответствующие устойчивому решению контактной задачи для высоких показателей градиентности. Показано, что адгезионный контакт между материалами с высоким показателем градиентности не разрушается при их удалении на любое расстояние друг от друга.

Ключевые слова: адгезия, градиентные среды, коэффициент Пуассона, индентирование, радиус контакта.

DOI: 10.21883/JTF.2021.11.51520.159-21

\section{Введение}

Функционально градиентные материалы - это материалы, свойства которых являются функцией координаты, т.е. изменяются в объеме образца $[1,2]$. К формированию градиентных характеристик могут приводить некоторые виды обработки, например, при сварке разнородных металлов сварной шов представляет переходную фазу, в которой свойства одного металла переходят в свойства другого. Градиентными свойствами обладает большое количество объектов, созданных природой. Это кости, иголки растений и животных, перья птиц, стебли растений, морские губки, хрящи суставов и т.д. Подавляющее большинство природных градиентных сред имеют биологическое происхождение и были сформированы в ходе эволюции. Это связано с тем, что очень часто органы из таких материалов лучше выполняют свои функции.

Градиентные материалы имеют большие перспективы использования в промышленности. Например, они применяются для создания покрытий, обеспечивающих плавную диссипацию механической энергии. Такие покрытия используются для защиты объектов от механических повреждений при их падении с большой высоты, либо для защиты от удара. Зная характеристики удара, можно теоретически рассчитать геометрическую форму объекта и градиентные характеристики материала, необходимого для того, чтобы возникающие при ударе в материале напряжения не превышали критически допустимых значений. В настоящее время проводятся как теоре- тические исследования указанных материалов [3-7], так и экспериментальные [8,9], большое внимание уделяется компьютерному моделированию $[10-12]$. В настоящей работе проведен анализ контактных свойств между несжимаемым индентором и полупространством, модуль упругости которого задается степенной зависимостью, которая характеризуется показателем градиентности $k$. Рассматриваются материалы с высоким показателем градиентности $k>1$, свойства которых принципиально отличаются от свойств материалов из классического диапазона $-1<k<1$.

\section{1. Диаграмма допустимых значений показателя градиентности}

Рассмотрим случай, в котором модуль упругости полупространства $E$ изменяется с его глубиной $z$ по степенному закону [13-15]

$$
E(z)=E_{0}\left(\frac{z}{c_{0}}\right)^{k},
$$

который проиллюстрирован на рис. 1 .

В подавляющем большинстве работ рассматривается интервал значений $0 \leq k<1[3,16,17]$, поскольку в этом интервале решение контактной задачи является устойчивым. ${ }^{1}$ Однако устойчивое решение может быть получено также и в некоторых других случаях. Например,

\footnotetext{
${ }^{1}$ Значение $k=0$ соответствует однородной среде с модулем упругости $E=E_{0}$.
} 


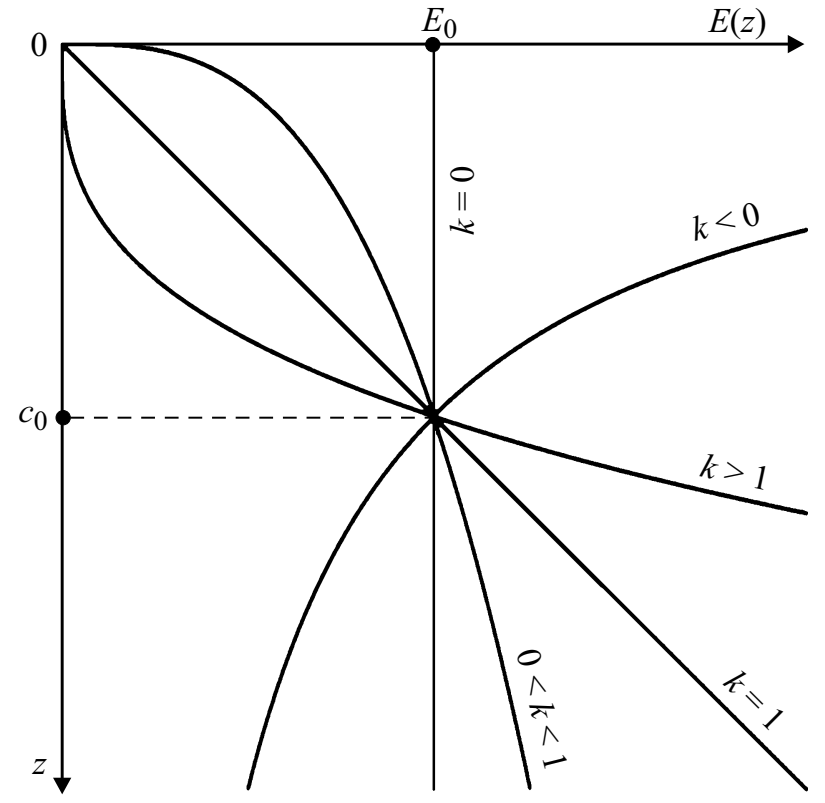

Рис. 1. Функциональная зависимость модуля упругости $E$ от глубины $z$ (1) при различных значениях показателя градиентности $k$.

в [18] показано, что для несжимаемых материалов с коэффициентом Пуассона $v=1 / 2$ решение контактной задачи является устойчивым в более широком интервале $0 \leq k<3$, причем ситуации $k<1$ и $k>1$ приводят к различным типам поведения исследуемой контактной системы. Например, в случае адгезионного контакта цилиндрического штемпеля с полупространством в случае $k<1$ при превышении критического расстояния между штемпелем и полупространством контакт мгновенно разрушается. Для значений показателя градиентности $k>1$ при удалении штемпеля от полупространства на величину, больше критической, радиус контакта начинает постепенно уменьшаться, но контакт никогда не разрушается полностью и существует при любом расстоянии между контактирующими телами $[18,19]$. Причины такого поведения кроются в значении производной

$$
\frac{\partial E(z)}{\partial z}=\frac{E_{0} k}{c_{0}^{k}} z^{k-1}
$$

в точке $z=0$, соответствующей началу индентирования. Рассмотрим случай $k>0$, в котором степенная зависимость (1) описывает нарастание модуля упругости с увеличением глубины индентирования $z$ от нулевого значения $E(z=0)=0$. При этом на характер разрушения контакта критическим образом влияет скорость нарастания модуля $E$ с увеличением координаты $z$ в точке $z=0$. Так, в интервале $0<k<1$ в точке $z=0$ происходит стремительное нарастание модуля сдвига, что отвечает значению $\partial E /\left.\partial z\right|_{z=0} \rightarrow \infty$ (см. также рис. 1). Поэтому материалы, характеризующиеся показателем градиентности $0<k<1$, начинают оказывать суще- ственное сопротивление индентированию сразу после возникновения контакта. В ситуации $k>1$ производная $\partial E /\left.\partial z\right|_{z=0}=0$, поэтому в начале индентирования сила реакции со стороны полупространства будет несущественной (рис. 1). В процессе разрушения адгезионного контакта описанные особенности приводят к тому, что в случае $k>1$ с увеличением расстояния между полупространством и индентором контакт никогда не разрушается, а лишь уменьшается его радиус $[18,19]$. Следует отметить, что в литературе рассматривается также интервал значений $-1<k<0$, отвечающий ситуации, когда модуль сдвига уменьшается с увеличением глубины $z[20,21]$. Все описанные выше случаи проиллюстрированы на рис. 1.

Целью настоящей работы является анализ расширенного диапазона значений параметра градиентности $k$, в котором могут существовать устойчивые решения задачи теории упругости. Проведем анализ на примере аксиально симметричного контакта с радиусом $a$. В задачах теории упругости часто вводится в рассмотрение понятие контактной жесткости. При индентировании в нормальном направлении контактная жесткость определяется как производная от нормальной силы $F$ по глубине индентирования $d$, т. е. $k_{N}=\mathrm{d} F / \mathrm{d} d$. Зная выражение для контактной жесткости, легко определить значение действующей силы, в зависимости от глубины индентирования. В случае нормального индентирования жесткого индентора в упругое полупространство с градиентными свойствами контактная жесткость определяется равенством [17]:

$$
k_{N}(a)=\frac{2 E_{0} h_{N}(k, v) a^{k+1}}{\left(1-v^{2}\right) c_{0}^{k}(k+1)},
$$

где функция $h_{N}(k, v)$ задается выражениями $[3,16,17]^{2}$

$$
\begin{gathered}
h_{N}(k, v)=\frac{2(1+k) \cos \left(\frac{k \pi}{2}\right) \Gamma\left(1+\frac{k}{2}\right)}{\sqrt{\pi} C(k, v) \beta(k, v) \sin \left(\frac{\beta(k, v) \pi}{2}\right) \Gamma\left(\frac{1+k}{2}\right)}, \\
C(k, v)=\frac{2^{1+k} \Gamma\left(\frac{3+k+\beta(k, v)}{2}\right) \Gamma\left(\frac{3+k-\beta(k, v)}{2}\right)}{\pi \Gamma(2+k)}, \\
\beta(k, v)=\sqrt{(1+k)\left(1-\frac{k v}{1-v}\right)},
\end{gathered}
$$

где Г(.) - гамма-функция [22].

Выше при обсуждении формулы (1) мы уже оговаривали интервалы значений параметра градиентности $k$, при которых может быть получено устойчивое решение контактной задачи. Устойчивыми решениями в этом понимании являются такие, которые обеспечивают положительные значения жесткости контакта $k_{N}$ (3).

\footnotetext{
2 При некоторых значениях параметров $k$ и $v$ функция $\beta(k, v)$ может быть мнимой величиной, но при этом результирующая функция $h_{N}(k, v)$ всегда принимает вещественные значения.
} 


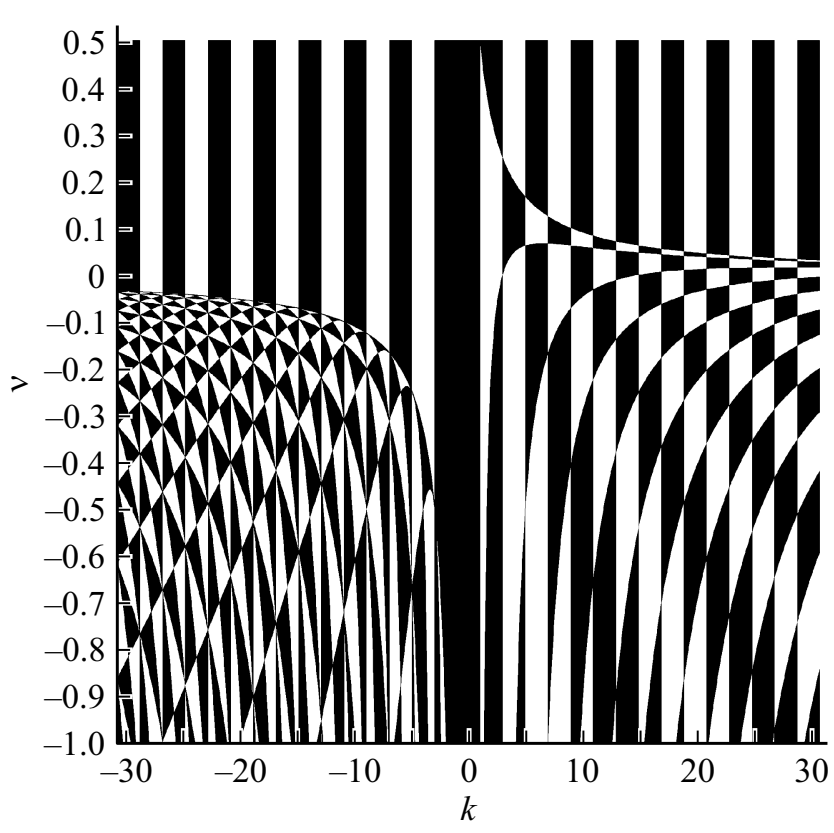

Pис. 2. Диаграмма, на которой закрашенные черным области отвечают положительным значениям контактной жесткости $k_{N}(3)$, светлые области отвечают значениям $k_{N}<0$.

При некоторых значениях параметров $k$ и $v$ функция $h_{N}(k, v)<0$, а это, согласно структуре соотношения (3), приводит к отрицательным значениям жесткости. Величины $k_{N}<0$ отвечают ситуации, в которой при индентировании в упругое полупространство сила его реакции совпадает с направлением индентирования. При этом полупространство не сопротивляется вдавливанию, а наоборот, стремится сразу после возникновения контакта втянуть в себя индентор, а такая ситуация не имеет физического смысла. Поэтому хотя аналитические решения контактных задач для упругого полупространства, градиентные свойства которого задаются уравнением (1), были опубликованы более 35 лет назад [13,14], в современной литературе до сих пор обсуждаются интервалы значений показателя градиентности $k$, соответствующие физически интерпретируемым результатам. В современных работах в этом направлении рассматривается интервал значений $-1<k<1$ [20,21]. В [18] показано, что для несжимаемых сред с коэффициентом Пуассона $v=1 / 2$ справедлив более широкий диапазон $-1<k<3$, причем при $k>1$ контактная система демонстрирует принципиально другие физические свойства $[18,19]$.

На рис. 2 показана рассчитанная диаграмма в координатах $k-v$, на которой закрашенные черным области отвечают положительным значениям функции $h_{N}(k, v)$ и соответственно положительной жесткости $k_{N}(3)^{3}$.

\footnotetext{
${ }^{3}$ Вертикальные чередующиеся полосы на диаграмме на рис. 2 связаны с изменением знака множителей $\cos (k \pi / 2)$ и $\sin (\beta \pi / 2)$ в выражении $h_{N}(k, v)(4)$.
}

Значения коэффициента Пуассона на диаграмме показаны в диапазоне $-1<v \leq 1 / 2$, который отвечает термодинамически устойчивым материалам [23]. Показатель градиентности на рис. 2 принимает значения на значительно более широком интервале $-31<k<31$ по сравнению с классическим диапазоном $-1<k<1$. На диаграмме классическому интервалу отвечает сплошная черная вертикальная полоса, поскольку на нем решения являются устойчивыми при любых значениях коэффициента Пуассона v. Более того, согласно диаграмме, положительные значения контактной жесткости $k_{N}$ реализуются на более широком интервале $-2<k<1$, вне зависимости от величины $v$. Поэтому классический интервал применения теории $-1<k<1$ может быть расширен слева до $-2<k<1$, без введения какихлибо ограничений. Из диаграммы также следует, что при $v=1 / 2$ показатель градиентности $k$ может принимать значения $-2<k<3$, обеспечивая при этом устойчивые решения, как это было продемонстрировано в работах $[18,19]$.

На диаграмме на рис. 2 мы показали область с отрицательным значением коэффициента Пуассона, поскольку такие специфические материалы (ауксетики) существуют в природе - это некоторые полимеры, бумага, кристаллический $\mathrm{Na}$ и другие [24]. Однако наиболее широкий класс материалов характеризуется положительными значениями $v>0$, поэтому область $v>0$ является более актуальной для реальных технических приложений. В дальнейшем исследовании остановимся на положительных значениях параметpa $v$. Соответствующая диаграмма приведена на рис. 3 для более узкого диапазона показателя градиентности $-2<k<31$.

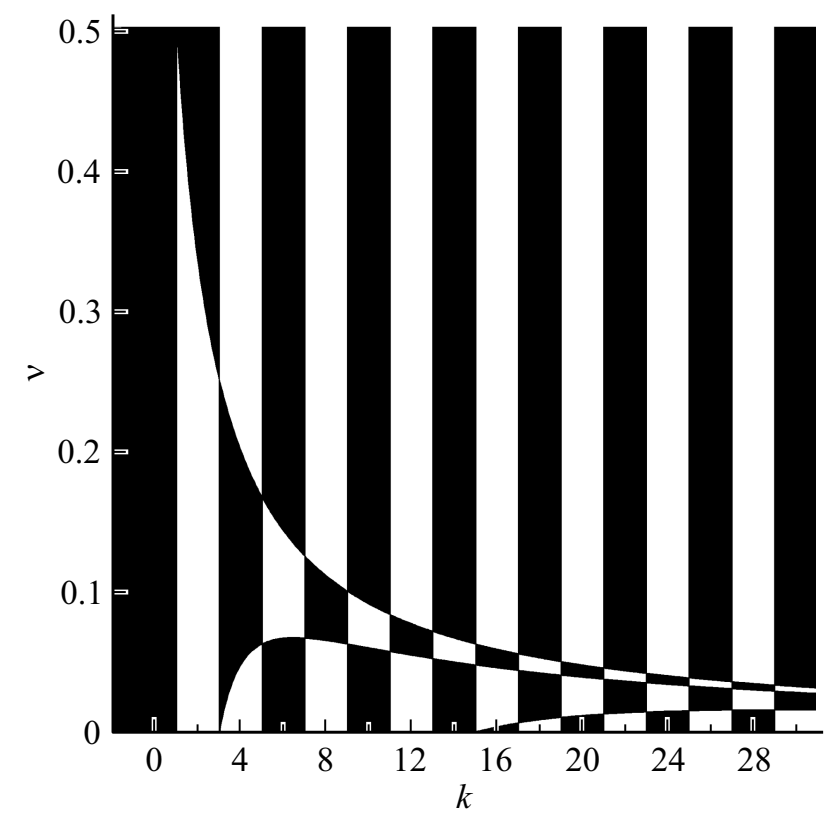

Рис. 3. Увеличенная часть диаграммы, которая приведена на рис. 2, для положительных значений коэффициента Пуассона $v$. 

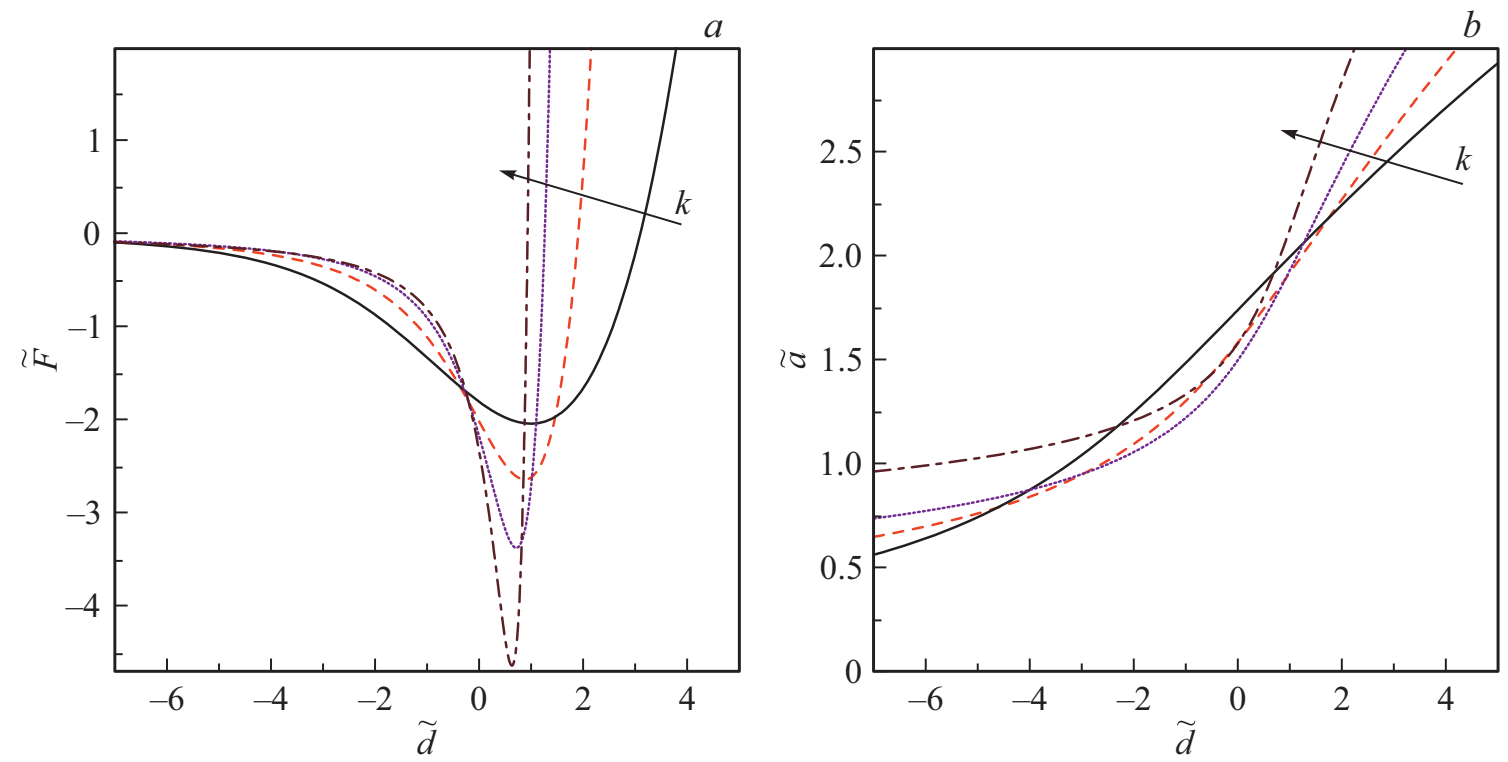

Рис. 4. $a$ - зависимости нормальной силы $\tilde{F}$ от глубины индентирования $\tilde{d}$, задающиеся формулами (5) и (6), построенные при значениях $v=0.1$ и $\tilde{c}_{0}=1.0$. Кривые, порядок которых указан стрелкой, соответствуют значениям показателя градиентности $k=3.1,4.9,7.1$ и $10.9 ; b-$ зависимости радиуса контакта $\tilde{a}$ от глубины индентирования $\tilde{d}$, соответствующие кривым, показанным на панели $a$.

\section{2. Свойства адгезионного контакта между телами с высоким показателем градиентности}

Приведенный в разд. 1 настоящей работы анализ расширяет диапазон применимости модели градиентных сред для описания контакта материалов с высокими показателями градиентности. В разд. 2 настоящей работы покажем, что свойства систем с высокими значениями $k$ могут качественно отличаться от свойств контактных систем из привычного диапазона $-1<k<1$. Соответствующий анализ будем проводить на основе адгезионного контакта. Адгезия играет большую роль в разнообразных технических приложениях, таких как склеивание поверхностей, пайка, сварка, нанесение покрытий, фасовка удобрений и многое другое [25]. При наличии сильно выраженного адгезионного взаимодействия между контактирующими поверхностями адгезионные процессы критическим образом влияют на контактное поведение. Поэтому выбор для дальнейшего анализа адгезионного контакта имеет прямое прикладное значение. Первой строгой теорией, описывающей адгезионный контакт, является теория JKR [26]. Подход JKR исходит из предположения, что расстояния, на которых действуют адгезионные силы, ничтожно малы по сравнению с геометрическими параметрами контакта - такими как глубина индентирования и радиус контакта. Подход JKR на сегодняшний день является наиболее часто используемым при описании экспериментальных результатов как в макроскопических инженерных контактах, так и в наноразмерных системах. Важно так же и то, что в подходе $\mathrm{JKR}$ существуют известные аналитические решения для градиентных сред, задающие зависимости нормальной силы от глубины индентирования, что упрощает проводимый далее анализ. Для изучения влияния показателя $k$ на свойства адгезионного контакта проанализируем классическое решение для контакта параболического несжимаемого индентора с радиусом кривизны $R$ с упругим полупространством, обладающим градиентными свойствами. В случае адгезионного взаимодействия типа JKR зависимость контактной силы от глубины индентирования задается соотношениями [16,25,27]:

$$
\begin{gathered}
\tilde{d}=\frac{3 \tilde{a}^{2}}{1+k}-4 \sqrt{\frac{\tilde{a}^{1-k} \tilde{c}_{0}^{k}\left(1-v^{2}\right)}{h(k, v)}} \\
\tilde{F}=\frac{3 h_{N}(k, v) \tilde{a}^{3+k}}{\tilde{c}_{0}^{k}(1+k)^{2}(3+k)\left(1-v^{2}\right)}-2 \sqrt{\frac{\tilde{a}^{3+k} h_{N}(k, v)}{(1+k)^{2} \tilde{c}_{0}^{k}\left(1-v^{2}\right)}},
\end{gathered}
$$

где $\tilde{d}, \tilde{a}, \tilde{F}$ и $\tilde{c}_{0}-$ безразмерные значения глубины индентирования, радиуса контакта, нормальной силы и параметра $c_{0}$, измеренные в единицах

$$
\begin{gathered}
F_{0}=\frac{3}{2} \pi R \Delta \gamma ; \quad a_{0}=\left(\frac{9 \pi R^{2} \Delta \gamma}{8 E_{0}}\right)^{1 / 3}, \\
d_{0}=\left(\frac{3 \pi^{2} R \Delta \gamma^{2}}{64 E_{0}^{2}}\right)^{1 / 3} .
\end{gathered}
$$

Размерные параметры определяются как $d=\tilde{d} \cdot d_{0}$, $a=\tilde{a} \cdot a_{0}, \quad F=\tilde{F} \cdot F_{0}$ и $c_{0}=\tilde{c}_{0} \cdot a_{0}$. Единицы измерения (7) отличаются от использованных ранее в [19]. Отличие состоит в том, что выражения (7) не содержат коэффициент Пуассона $v$, поскольку в настоящей работе 
анализируется влияние параметров $k$ и $v$ на контактные свойства. Поэтому коэффициент Пуассона $v$ входит непосредственно в формулы (5) и (6).

На рис. 4, а показаны рассчитанные по формулам (5) и (6) зависимости нормальных сил от глубины индентирования при различных значениях показателя градиентности $k$. В отличие от контакта без адгезии адгезионный контакт существует также и при отрицательных значениях глубины индентирования $\tilde{d}$ (когда индентор приподнят над полупространством), поскольку за счет адгезии контактирующие материалы „прилипают“ друг к другу. Для разрушения такого контакта необходимо приложить дополнительную силу. Адгезионная составляющая нормальной силы всегда направлена против силы реакции упругого полупространства, которое сопротивляется вдавливанию индентора. Результирующая контактная сила $\tilde{F}$, показанная на рис. 4, $a$, представляет разницу между силой реакции полупространства и силой адгезии. В случае, когда адгезионная компонента выше силы реакции, результирующая сила $\tilde{F}<0$. За счет адгезии происходит увеличение радиуса контакта $a$ (рис. $4, b$ ). Поэтому радиус контакта при наличии адгезии всегда больше, чем радиус контакта в той же системе, но без адгезионного взаимодействия между контактирующими телами.

Из рис. 4 следует, что случай $k>1$ соответствует ситуации, когда вне зависимости от величины удаления индентора от полупространства адгезионный контакт никогда не разрушается полностью, поскольку радиус контакта $a$ всегда больше нуля. Качественно результаты, продемонстрированные на рис. 4, повторяют результаты работы [19], в которой рассматривался случай несжимаемых градиентных сред с коэффициентом Пуассона $v=1 / 2$ в области показателя градиентности $1<k<3$. Из рис. 4, $a$ следует, что при фиксированном значении коэффициента Пуассона с увеличением показателя градиентности $k$ увеличивается абсолютное значение адгезионной силы $\tilde{F}<0$, т.е. с ростом показателя $k$ прочность контакта увеличивается. В [16] приведены аналитические выражения характерных величин для рассматриваемой задачи, которые были получены при анализе уравнений (5) и (6). Все выражения из работы [16] остаются справедливыми и для предлагаемого в настоящей работе исследования, поскольку при их выводе на показатель $k$ не вводились никакие ограничения. В частности, выражение для максимальной адгезионной силы, возникающей при разрушении контакта, при использовании единиц измерения (7) запишется в виде [16]

$$
\tilde{F}_{c}=-\frac{k+3}{3} \text {. }
$$

Однако при этом стоит учитывать, что некоторые критические величины, приведенные в [16], для диапазона $k>1$ теряют изначально вложенное в них содержание. К примеру, критическая глубина индентирования $d_{c}$, при которой контакт разрушается, таковой более не является, поскольку, согласно проведенному анализу в области $k>1$, адгезионный контакт никогда полностью не разрушается. Это видно из рис. $4, b$, на котором показаны зависимости радиуса контакта $\tilde{a}$ от глубины индентирования $\tilde{d}$, соответствующие кривым, показанным на рис. 4, $a$ (см. также работу [19]).

\section{Заключение}

В работе исследуются контактные свойства градиентных материалов с высоким показателем градиентности с наличием адгезионного взаимодействия между контактирующими телами. Построена диаграмма в координатах коэффициент Пуассона - показатель градиентности, на которой указаны области существования устойчивых решений контактной задачи. Показано, что высокие значения показателя градиентности $k>1$ приводят к тому, что после возникновения адгезионного контакта он никогда не разрушается, вне зависимости от величины расстояния между контактирующими телами. В работе рассмотрен только нормальный контакт, но аналогичное исследование может быть обобщено на случай отрыва индентора под произвольным углом, т.е. при наличии тангенциального движения. Такое исследование мы планируем провести в последующих работах.

\section{Финансирование работы}

Работа выполнена при финансовой поддержке Немецкого научно-исследовательского сообщества (Deutsche Forschungsgemeinschaft), проект PO 810-55-3.

\section{Конфликт интересов}

Авторы заявляют, что у них нет конфликта интересов.

\section{Список литературы}

[1] Y. Miyamoto, W.A. Kaysser, B.H. Rabin, A. Kawasaki, R.G. Ford. Functionally Graded Materials: Design, Processing and Applications (Springer Science+Business Media, LLC, NY., 1999)

[2] A. Gupta, T. Mohammad. Prog. Aerosp. Sci., 79, 1 (2015). DOI: 10.1016/j.paerosci.2015.07.001

[3] F. Jin, X. Guo, W. Zhang. J. Appl. Mech., 80, 061024 (2013). DOI: $10.1115 / 1.4023980$

[4] I. Argatov, A. Iantchenko. Q. J. Mech. Appl. Math., 72, 197 (2019). DOI: 10.1093/qjmam/hbz002

[5] J. Aboudi, M.-J. Pindera, S.M. Arnold. Compos. B Eng., 30, 777 (1999). DOI: 10.1016/S1359-8368(99)00053-0

[6] E. Willert. FU Mech. Eng., 16, 9 (2018). DOI: $10.22190 /$ FUME171121003W

[7] I.A. Lyashenko, V.N. Borysiuk, V.L. Popov. FU Mech. Eng., 18, 245 (2020). DOI: 10.22190/FUME200129020L 
[8] M. Hill, R. Carpenter, G. Paulino, Z. Munir, J. Gibeling. Fracture Testing of a Layered Functionally Graded Material, in Fracture Resistance Testing of Monolithic and Composite Brittle Materials, ed. J. Salem, G. Quinn, and M. Jenkins (West Conshohocken, PA: ASTM International, 2002), p. 169. DOI: $10.1520 / \mathrm{STP} 10478 \mathrm{~S}$

[9] C.-E. Rousseau, V.B. Chalivendra, H.V. Tippur, A. Shukla. Exp. Mech., 7, 845 (2010). DOI: 10.1007/s11340-010-9381-Z

[10] V.L. Popov, R. Pohrt, Q. Li. Friction, 5, 308 (2017). DOI: $10.1007 / \mathrm{s} 40544-017-0177-3$

[11] E. Martinez-Paneda, R. Gallego. Int. J. Mech. Mater. Des., 11, 405 (2015). DOI: 10.1007/s10999-014-9265-y

[12] M. Scaraggi, D. Comingio. Int. J. Solids Struct., 125, 276 (2017). DOI: 10.1016/j.jijsolstr.2017.06.008

[13] J.R. Booker, N.P. Balaam, E.H. Davis. Int. J. Numer. Anal. Methods. Geomech., 9, 353 (1985).

DOI: 10.1002/nag.1610090405

[14] J.R. Booker, N.P. Balaam, E.H. Davis. Int. J. Numer. Anal. Methods. Geomech., 9, 369 (1985).

DOI: $10.1002 /$ nag.1610090406

[15] A.E. Giannakopoulos, S. Suresh. Int. J. Solids Struct., 34, 2357 (1997). DOI: 10.1016/S0020-7683(96)00171-0

[16] M. Hess, V.L. Popov. FU Mech. Eng., 14, 251 (2016). DOI: $10.22190 /$ FUME1603251H

[17] M. Hess. Int. J. Eng. Sci., 104, 20 (2016).

DOI: 10.1016/j.ijengsci.2016.04.009

[18] V.L. Popov. Phys. Mesomech., 21, 76 (2018).

DOI: $10.1134 / \mathrm{S} 1029959918010101$

[19] Я.А. Ляшенко, В.Л. Попов. ЖТФ, 90 (5), 760 (2020). DOI: $10.21883 /$ JTF.2020.05.49176.140-18 [I.A. Lyashenko, V.L. Popov. Tech. Phys., 65 (10), 1695 (2020). DOI: $10.1134 / \mathrm{S} 1063784220100126]$

[20] Q. Li, V.L. Popov. Comput. Mech., 61, 319 (2018). DOI: $10.1007 / \mathrm{s} 00466-017-1461-9$

[21] D. Lee, J.R. Barber, M.D. Thouless. Int. J. Eng. Sci., 47, 1274 (2009). DOI: 10.1016/j.ijengsci.2008.08.005

[22] А.Ф. Никифоров, В.Б. Уваров. Специальные функции математической физики (Наука, М., 1978)

[23] Л.Д. Ландау, Е.М. Лифшиц. Теория упругости (Наука, М., 1965), изд. 3-е, испр. и доп.

[24] R. Lakes. Science., 235, 1038 (1987). DOI: $10.1126 /$ science. 235.4792 .1038

[25] Handbook of Adhesion Technology [Ed. by L.F.M. da Silva, A. Öchsner, R.D. Adams], 2-nd Ed. (Springer, Berlin, 2018). DOI: $10.1007 / 978-3-319-55411-2$

[26] K.L. Johnson, K. Kendall, A.D. Roberts. Proc. Royal Soc. Lond. A, 324, 301 (1971). DOI: 10.1098/rspa.1971.0141

[27] K.L. Johnson. Proc. R. Soc. Lond. A, 453, 163 (1997). DOI: $10.1098 /$ rspa.1997.0010 